\title{
Association between different growth curve definitions of overweight and obesity and cardiometabolic risk in children
}

\author{
Lisa Kakinami PhD, Mélanie Henderson MD, Edgard E. Delvin PhD, Emile Levy MD PhD,
} Jennifer O’Loughlin PhD, Marie Lambert MD, Gilles Paradis MD

\begin{abstract}
Background: Overweight and obesity in young people are assessed by comparing body mass index (BMI) with a reference population. However, two widely used reference standards, the Centers for Disease Control and Prevention (CDC) and the World Health Organization (WHO) growth curves, have different definitions of overweight and obesity, thus affecting estimates of prevalence. We compared the associations between overweight and obesity as defined by each of these curves and the presence of cardiometabolic risk factors.
\end{abstract}

Methods: We obtained data from a populationrepresentative study involving 2466 boys and girls aged 9, 13 and 16 years in Quebec, Canada. We calculated BMI percentiles using the CDC and WHO growth curves and compared their abilities to detect unfavourable levels of fasting lipids, glucose and insulin, and systolic and diastolic blood pressure using receiver operating characteristic curves, sensitivity, specificity and kappa coefficients.
Results: The $z$ scores for BMI using the WHO growth curves were higher than those using the CDC growth curves (0.35-0.43 v. 0.12$0.28, p<0.001$ for all comparisons). The WHO and CDC growth curves generated virtually identical receiver operating characteristic curves for individual or combined cardiometabolic risk factors. The definitions of overweight and obesity had low sensitivities but adequate specificities for cardiometabolic risk. Obesity as defined by the WHO or CDC growth curves discriminated cardiometabolic risk similarly, but overweight as defined by the WHO curves had marginally higher sensitivities (by $0.6 \%-8.6 \%$ ) and lower specificities (by $2.6 \%-4.2 \%$ ) than the CDC curves.

Interpretation: The WHO growth curves show no significant discriminatory advantage over the CDC growth curves in detecting cardiometabolic abnormalities in children aged 9-16 years.
$\mathrm{P}$ ediatric obesity is associated with dyslipidemia, insulin resistance and elevated blood pressure..$^{1-6}$ Thus, accurately identifying children with obesity is crucial for clinical management and public health surveillance.

Lipid screening is recommended for young people who are overweight, ${ }^{7.8}$ but studies show that estimates of the prevalence of overweight and obesity are 1\%-7\% lower using the growth curves of the Centers for Disease Control and Prevention (CDC) versus those of the World Health Organization (WHO).$^{9-11}$ Although the CDC and WHO definitions of overweight and obesity both use approximations of overweight and obese values of body mass index (BMI) when children reach 19 years of age, the CDC growth curves use data from more recent samples of young people. ${ }^{12,13}$ Given the recent rise in the prevalence of obesity among young people, using a heavier reference population may lead to fewer children being identified as overweight and obese, and an identical BMI value may not trigger a clinical investigation. ${ }^{7}$ The Canadian Paediatric Society, in collaboration with the College of Family Physicians of Canada, Dietitians of Canada and Community Health Nurses of Canada, recently recommended that physicians switch from the CDC to the WHO growth curves for monitoring growth for Canadian children aged 5-19 years. ${ }^{14}$ This is a major change for health providers caring for the estimated 8 million children in Canada. ${ }^{15}$

Understanding how using the different growth curves affects the identification of adverse cardiometabolic risk profiles is essential for the appropriate management of overweight and obesity among young people. Thus, our objectives were to assess whether the association between BMI percentiles and cardiometabolic risk differs between the definitions of
Competing interests: None declared.

This article has been peer reviewed.

Correspondence to: Lisa Kakinami, lisa.kakinami@mcgill.ca

CMAJ 2012. DOI:10.1503 /cmaj.110797 
overweight and obesity based on the WHO and CDC growth curves, and to compare the sensitivity and specificity of these definitions in detecting cardiometabolic risk.

\section{Methods}

We used the study population from the Québec Child and Adolescent Health and Social Survey (QCAHS) for our analysis. The full details of the study and its data collection have been previously published. ${ }^{16}$ Briefly, the survey was a crosssectional, multistage, stratified, representative sample of young people aged 9, 13 and 16 years in the province of Quebec in 1999. Children from very remote areas, small schools or specialty schools (e.g., schools primarily serving children with disabilities or native/Aboriginal populations) were excluded $(<3 \%$ of the target population). The sampling frame consisted of three levels of clustered data: regional clusters, schools clustered within regions and students clustered within schools. Independent samples were selected within each age group from each school. The study was approved by the ethics committees of the Institut de la Statistique du Québec, the Ministère de l'Education du Québec and the Centre Hospitalier Universitaire Sainte-Justine. Informed consent and assent were provided by parents and participating children.

\section{Cardiometabolic measures}

We collected fasting venous blood samples between 8 am and $10 \mathrm{am}$, which we placed on ice until analysis. We centrifuged the samples within 45 minutes of collection, transported them on dry ice and stored them at $-80^{\circ} \mathrm{C}$. We analyzed the samples for total cholesterol, highdensity lipoprotein (HDL) cholesterol, triglyceride and glucose levels. All analyses were done at the Department of Clinical Biochemistry at Sainte-Justine using the standardized guidelines of the International Federation of Clinical Chemistry. ${ }^{17,18}$ We calculated the level of low-density lipoprotein (LDL) cholesterol using the Friedewald equation. ${ }^{19}$ We measured insulin levels using the ultrasensitive insulin kit on the Access immunoassay system. We measured participants' systolic and diastolic blood pressures after a 5-minute rest and at least 30 minutes after a light meal, according to standardized procedures..$^{20}$ We used the mean of the last two of three consecutive measurements of blood pressure taken at one-minute intervals for our analysis.

We categorized the levels of lipids and metabolic risk factors as either normal or unfa- vourable (borderline and high) according to pediatric cut-off values from the American Heart Association and the American Academy of Pediatrics. ${ }^{21}$ However, because there are no specific recommendations for HDL cholesterol or triglycerides, we categorized them as recommended in the literature. ${ }^{22}$ We defined unfavourable lipid levels as a total cholesterol level of $4.4 \mathrm{mmol} / \mathrm{L}$ or more, an HDL cholesterol level of less than $1.0 \mathrm{mmol} / \mathrm{L}$, an LDL cholesterol level of $2.6 \mathrm{mmol} / \mathrm{L}$ or more, and triglyceride levels of $1.7 \mathrm{mmol} / \mathrm{L}$ or more. We defined unfavourable metabolic factors as an insulin level of $38 \mathrm{pmol} / \mathrm{L}$ or more for children aged 9 years, or $60 \mathrm{pmol} / \mathrm{L}$ or more for children aged 13 and 16 years, and a blood glucose level of $5.6 \mathrm{mmol} / \mathrm{L}$ or more. We defined unfavourable blood pressure as at or above the 90th percentile for age-, sex- and height-adjusted blood pressure according to the National High Blood Pressure Education Program..$^{23}$

\section{Anthropometric measures}

We measured the weight and height of each participant twice using a calibrated spring scale (weight) and standard measuring tape (height). Participants wore light, indoor clothing and no shoes while their measurements were taken. If we saw a difference in weight of $0.2 \mathrm{~kg}$ or more, or a difference in height of $0.5 \mathrm{~cm}$ or more, a third measurement was taken. We used the average of the two closest measurements for our analysis. We calculated BMI using the standard formula (weight/height ${ }^{2}$, where weight is measured in kilograms, and height is measured in metres). Using the CDC growth curves, overweight is defined as a BMI at or above the 85 th percentile and below the 95th percentile, and obesity is defined as a BMI at or above the 95th percentile. ${ }^{12}$ Using the WHO growth curves, overweight is defined as a BMI at or above the 85th percentile and below the 97.7th percentile, and obesity is defined as a BMI at or above the 97.7th percentile. ${ }^{13}$

\section{Statistical analysis}

We stratified all analyses by sex. We used unadjusted models with no adjustment for covariates to assess the areas under the curves for continuous BMI percentiles, continuous BMI $z$ scores, separate binary categories for overweight and obese, and a single binary category for overweight or obese. Our results were consistent across all parameters of BMI, thus the areas under the curves from continuous BMI percentiles are uniformly presented. The area under the curve can be interpreted as the probability of a child's BMI percentile accurately detecting a 
Table 1: Characteristics of the 2466 participants of the study and their households, by sex and age

\begin{tabular}{|c|c|c|c|c|c|c|}
\hline \multirow[b]{3}{*}{ Characteristic } & \multicolumn{6}{|c|}{ No. $(\%)^{*}$} \\
\hline & \multicolumn{3}{|c|}{ Boys } & \multicolumn{3}{|c|}{ Girls } \\
\hline & $\begin{array}{c}\text { 9-year-olds } \\
n=381\end{array}$ & $\begin{array}{c}\text { 13-year-olds } \\
n=416\end{array}$ & $\begin{array}{c}16 \text {-year-olds } \\
n=407\end{array}$ & $\begin{array}{c}\text { 9-year-olds } \\
n=398\end{array}$ & $\begin{array}{c}13 \text {-year-olds } \\
n=400\end{array}$ & $\begin{array}{c}16 \text {-year-olds } \\
n=464\end{array}$ \\
\hline \multicolumn{7}{|l|}{ Household } \\
\hline \multicolumn{7}{|l|}{ Annual income, \$ } \\
\hline$<20000$ & $50(13.1)$ & $56(13.5)$ & 32 (7.9) & $53(13.3)$ & $57(14.3)$ & $48(10.3)$ \\
\hline 20 000-39999 & $82(21.5)$ & $88(21.1)$ & $85(20.9)$ & $101(25.4)$ & $71(17.7)$ & $89(19.2)$ \\
\hline $40000-59999$ & $94(24.7)$ & $85(20.4)$ & $98(24.1)$ & $97(24.4)$ & $101(25.2)$ & $113(24.3)$ \\
\hline$\geq 60000$ & $109(28.6)$ & $124(29.8)$ & $123(30.2)$ & $91(22.9)$ & $122(30.5)$ & $135(29.1)$ \\
\hline Unknown & $46(12.1)$ & $63(15.1)$ & 69 (16.9) & $56(14.1)$ & $49(12.2)$ & $79(17.0)$ \\
\hline \multicolumn{7}{|l|}{ Relative incomet } \\
\hline Very poor & $24 \quad(6.3)$ & $25 \quad(6.0)$ & 7 (1.7) & $16(4.0)$ & $24 \quad(6.0)$ & $20 \quad(4.3)$ \\
\hline Poor & $40(10.5)$ & $41 \quad(9.9)$ & 35 (8.6) & $56(14.1)$ & $44(11.0)$ & $37 \quad(8.0)$ \\
\hline Medium & $111(29.1)$ & $110(26.4)$ & $94(23.1)$ & $109(27.4)$ & $97(24.3)$ & $110(23.7)$ \\
\hline High & $108(28.3)$ & $113(27.2)$ & $137(33.7)$ & $122(30.6)$ & $124(31.0)$ & $149(32.1)$ \\
\hline Very high & $52(13.6)$ & $64(15.4)$ & $64(15.7)$ & $39 \quad(9.8)$ & $62(15.5)$ & 69 (14.9) \\
\hline Unknown & $46(12.1)$ & $63(15.1)$ & $70(17.2)$ & $56(14.1)$ & $49(12.2)$ & $79(17.0)$ \\
\hline \multicolumn{7}{|l|}{ Family } \\
\hline $\begin{array}{l}\text { Intact two-parent } \\
\text { household }\end{array}$ & $246(64.6)$ & $252(60.6)$ & $248(60.9)$ & $250(62.8)$ & $268(67.0)$ & $287(61.8)$ \\
\hline $\begin{array}{l}\text { Blended or single- } \\
\text { parent household }\end{array}$ & $97(25.4)$ & $118(28.4)$ & $104(25.5)$ & $106(26.6)$ & $100(25.0)$ & $122(26.3)$ \\
\hline Unknown & $38(10.0)$ & $46(11.0)$ & $55(13.5)$ & $42(10.6)$ & 32 (8.0) & $55(11.8)$ \\
\hline \multicolumn{7}{|l|}{$\begin{array}{l}\text { Parental level of } \\
\text { education }\end{array}$} \\
\hline $\begin{array}{l}\text { Less than high school, } \\
\text { one or both parents }\end{array}$ & $103(27.0)$ & $128(30.8)$ & $117(28.7)$ & $114(28.6)$ & $110(27.5)$ & $143(30.8)$ \\
\hline $\begin{array}{l}\text { High school graduate } \\
\text { or higher education, } \\
\text { both parents }\end{array}$ & $208(54.6)$ & $195(46.9)$ & $189(46.4)$ & $193(48.5)$ & $213(53.3)$ & $223(48.1)$ \\
\hline Unknown & $82(21.5)$ & $90(21.6)$ & $94(23.1)$ & $73(18.3)$ & $70(17.5)$ & $93(20.0)$ \\
\hline \multicolumn{7}{|l|}{$\begin{array}{l}\text { Self-identified } \\
\text { ethnocultural group }\end{array}$} \\
\hline $\begin{array}{l}\text { Canadian (French or } \\
\text { English) }\end{array}$ & $279(73.2)$ & $296(71.1)$ & $293(72.0)$ & 294 (73.9) & $293(73.3)$ & $333(71.8)$ \\
\hline Other & $20 \quad(5.2)$ & $30 \quad(7.2)$ & $20(4.9)$ & $31 \quad(7.8)$ & $37 \quad(9.3)$ & $38 \quad(8.2)$ \\
\hline Unknown & $82(21.5)$ & $90(21.6)$ & $94(23.1)$ & $73(18.3)$ & $70(17.5)$ & $93(20.0)$ \\
\hline \multicolumn{7}{|l|}{ Participant } \\
\hline $\begin{array}{l}\text { Age, mo, mean } \\
\text { (SD; range) }\end{array}$ & $\begin{array}{c}112.8 \\
(3.6 ; 106.0-121.0)\end{array}$ & $\begin{array}{c}160.6 \\
(3.6 ; 154.0-169.0)\end{array}$ & $\begin{array}{c}196.9 \\
(3.4 ; 190.0-205.0)\end{array}$ & $\begin{array}{c}112.7 \\
(3.6 ; 106.0-121.0)\end{array}$ & $\begin{array}{c}160.4 \\
(3.7 ; 154.0-169.0)\end{array}$ & $\begin{array}{c}196.9 \\
(3.5 ; 190.0-205.0)\end{array}$ \\
\hline $\begin{array}{l}\text { Height, cm, mean } \\
\text { (SD; range) }\end{array}$ & $\begin{array}{c}135.7 \\
(6.1 ; 120.0-155.7)\end{array}$ & $\begin{array}{c}160.0 \\
(9.0 ; 124.5-186.3)\end{array}$ & $\begin{array}{c}174.8 \\
(7.2 ; 153.0-196.2)\end{array}$ & $\begin{array}{c}135.2 \\
(6.6 ; 109.9-155.9)\end{array}$ & $\begin{array}{c}158.9 \\
(6.2 ; 123.3-174.0)\end{array}$ & $\begin{array}{c}162.6 \\
(5.9 ; 141.5-179.7)\end{array}$ \\
\hline $\begin{array}{l}\text { Weight, kg, mean } \\
\text { (SD; range) }\end{array}$ & $\begin{array}{c}32.3 \\
(7.5 ; 18.2-74.0)\end{array}$ & $\begin{array}{c}52.4 \\
(12.7 ; 21.0-117.0)\end{array}$ & $\begin{array}{c}68.6 \\
(13.3 ; 42.7-139.5)\end{array}$ & $\begin{array}{c}32.2 \\
(8.3 ; 20.0-85.7)\end{array}$ & $\begin{array}{c}52.8 \\
(12.6 ; 27.5-124.0)\end{array}$ & $\begin{array}{c}58.7 \\
(11.6 ; 36.0-115.0)\end{array}$ \\
\hline $\begin{array}{l}\mathrm{BMI}, \mathrm{kg} / \mathrm{m}^{2}, \text { mean } \\
\text { (SD; range) }\end{array}$ & $\begin{array}{c}17.4 \\
(3.1 ; 12.6-35.0)\end{array}$ & $\begin{array}{c}20.3 \\
(3.8 ; 13.5-40.1)\end{array}$ & $\begin{array}{c}22.4 \\
(3.9 ; 16.0-42.3)\end{array}$ & $\begin{array}{c}17.4 \\
(3.5 ; 11.1-40.9)\end{array}$ & $\begin{array}{c}20.8 \\
(4.4 ; 11.2-43.4)\end{array}$ & $\begin{array}{c}22.2 \\
(4.0 ; 15.6-43.7)\end{array}$ \\
\hline
\end{tabular}


normal or unfavourable level of risk: an area under the curve of 0.50 is considered uninformative and detecting cardiometabolic risk factors no better than chance; an area under the curve greater than 0.80 is considered good. ${ }^{24,25}$

Because the effects of obesity on cardiometabolic risk may vary between people, we also assessed the ability of the growth curves to detect any single, any cluster of two, or three or more (v. none) unfavourable factors from among HDL cholesterol, LDL cholesterol, triglycerides, systolic and diastolic blood pressure, glucose and insulin. We excluded total cholesterol owing to its high correlation with LDL cholesterol.

We used kappa coefficients to determine the level of agreement between the two growth curves, and the Youden index to identify the optimal sex-specific cut-offs for BMI percentile for cardiometabolic abnormalities. ${ }^{26}$ We identified the sensitivities and specificities of the CDC and WHO definitions for detecting unfavourable levels of cardiovascular risk factors as acceptable if they were higher than $70 \% .{ }^{24}$ All analyses accounted for the nonindependence between observations: we analyzed descriptive characteristics using paired $t$ tests and Friedman tests; the areas under the receiver operating characteristic curves incorporated a smaller standard error; we tested the sensitivity and specificity between growth curves using the McNemar $\chi^{2}$ test; and we calculated confidence intervals for the differences using a continuity correction. ${ }^{27-30}$ We used SAS software to create receiver operating characteristic curves, but we compared them using a macro available online (www.medicine.mcgill.ca/epidemiology /hanley/software/delong_sas.html). ${ }^{27,28}$

\section{Results}

Of the 4643 participants in the study, 2475 provided blood samples, blood pressure and anthropometric measures, and were included in this analysis. Nine-year-old English-speaking children and very physically active 16 -year-old children were less likely to provide blood samples (data not shown). Although nonparticipants had a lower mean BMI than participants (19.7 v. 20.2, $p=0.001$ ), their BMI percentiles and $z$ scores were similar, and no other statistically significant differences were noted between the

Table 2: Cardiometabolic risk factors among the 2466 children involved in the study, by sex and age

\begin{tabular}{|c|c|c|c|c|c|c|}
\hline \multirow{2}{*}{ Risk factor } & \multicolumn{6}{|c|}{ No. $(\%) *$} \\
\hline & \multicolumn{3}{|c|}{ Boys } & \multicolumn{3}{|c|}{ Girls } \\
\hline \multicolumn{7}{|l|}{ Cardiometabolic abnormality } \\
\hline HDL cholesterol level $<1.0 \mathrm{mmol} / \mathrm{L}$ & $21 \quad(5.5)$ & $39 \quad(9.4)$ & $98(24.1)$ & 18 (4.5) & 38 (9.5) & $41 \quad(8.8)$ \\
\hline LDL cholesterol $\geq 2.6 \mathrm{mmol} / \mathrm{L}$ & $107(28.1)$ & $84(20.2)$ & $71(17.4)$ & $155(38.9)$ & $115(28.8)$ & $155(33.4)$ \\
\hline Triglyceride level $\geq 1.7 \mathrm{mmol} / \mathrm{L}$ & $9 \quad(2.4)$ & $15 \quad(3.6)$ & $24 \quad(5.9)$ & $18 \quad(4.5)$ & $18(4.5)$ & $29(6.3)$ \\
\hline Blood pressure $\geq 90$ th percentilet & $n=378$ & $n=412$ & $n=407$ & $n=394$ & $n=399$ & $n=463$ \\
\hline Systolic & $46(12.1)$ & $73(17.7)$ & $124(30.5)$ & $40(10.2)$ & $82(20.6)$ & $84(18.1)$ \\
\hline \multirow[t]{2}{*}{ Blood glucose level $\geq 5.6 \mathrm{mmol} / \mathrm{L}$} & $n=381$ & $n=416$ & $n=407$ & $n=398$ & $n=400$ & $n=464$ \\
\hline & $38(10.0)$ & $85(20.4)$ & $92(22.6)$ & $25(6.3)$ & $60(15.0)$ & $37(8.0)$ \\
\hline Clustered risk factorsł & $n=381$ & $n=416$ & $n=407$ & $n=398$ & $n=400$ & $n=464$ \\
\hline 0 & $177(46.5)$ & $180(43.3)$ & $138(33.9)$ & $158(39.7)$ & $142(35.5)$ & $168(36.2)$ \\
\hline 1 cardiometabolic abnormality & $140(36.7)$ & $137(32.9)$ & $135(33.2)$ & $156(39.2)$ & $135(33.8)$ & $160(34.5)$ \\
\hline 2 cardiometabolic abnormalities & $46(12.1)$ & $62(14.9)$ & $78(19.2)$ & $59(14.8)$ & $74(18.5)$ & $98(21.1)$ \\
\hline$\geq 3$ cardiometabolic abnormalities & $18(4.7)$ & $37(8.9)$ & $56(13.8)$ & $25(6.3)$ & $49(12.3)$ & $38(8.2)$ \\
\hline \multicolumn{7}{|c|}{$\begin{array}{l}\text { Note: } \mathrm{HDL}=\text { high density lipoprotein cholesterol, } \mathrm{LDL}=\text { low density lipoprotein cholesterol. } \\
\text { *Percentages may not add up to } 100 \% \text { owing to rounding. } \\
+\geq 90 \text { th percentile for age, height and sex, as defined by the National High Blood Pressure Education Program. } \\
\text { fOne or more of the cardiometabolic risk factors as defined in the Table. Missing values were assumed to be normal. }\end{array}$} \\
\hline
\end{tabular}


two groups. In addition, we excluded nine participants for whom height and/or weight data were missing, resulting in a final sample size of 2466 children.

Our sample included 1204 boys (381 were 9 years old, 416 were 13 years old, 407 were 16 years old) and 1262 girls (398 were 9 years old, 400 were 13 years old, 464 were 16 years old). The descriptive characteristics and cardiometabolic profile of this population have been previously published and are briefly presented in Tables 1 and 2. ${ }^{16,22}$ We noted sex differences in cardiometabolic risk factors - fewer boys had unfavourable total cholesterol and insulin levels compared with girls of the same age, and fewer girls had unfavourable blood glucose levels compared with boys of the same age (Table 2). Regardless of sex or age, our estimates of the prevalence of unfavourable total cholesterol, LDL cholesterol and insulin levels were 20\%$30 \%$; we estimated the prevalence of unfavourable triglyceride level to be less than $10 \%$. The mean BMI percentiles and BMI $z$ scores as calculated using the WHO growth curves were significantly higher than those calculated using the CDC growth curves (Table 3).
The areas under the curves ranged from uninformative $(0.42)$ to good $(0.89)$, and were generally better able to detect unfavourable cardiometabolic factors with low prevalence such as triglyceride and HDL cholesterol levels, diastolic blood pressure, or clusters of three or more cardiometabolic risk factors (Table 4). We noted differences across sex and age: although the areas under the curves for unfavourable triglyceride levels among boys were greater than 0.80 , they were less than 0.65 for 13- and 16-year-old girls, and the areas under the curves were generally larger for younger age groups. Our results were unchanged when we reanalyzed the data using the definitions of the American Academy of Pediatrics for high total cholesterol $(\geq 5.2 \mathrm{mmol} / \mathrm{L})$ and LDL cholesterol $(\geq 3.4 \mathrm{mmol} / \mathrm{L}$ ) levels (data not shown). The areas under the curves were not significantly different from one another (all $p>$ 0.05 ). Our results were unchanged when we compared the WHO and CDC definitions of overweight and obese (data not shown).

The Youden index showed a wide range of cut-offs for optimal BMI percentiles based on the cardiometabolic risk factors of interest (Table 5), most of which were lower than the

Table 3: Comparison of the anthropometric characteristics of children according to whether the CDC or WHO growth curves are used as the reference

\begin{tabular}{|c|c|c|c|c|c|c|c|c|c|}
\hline Characteristic & $C D C$ & WHO & $\begin{array}{c}p \\
\text { value }\end{array}$ & $C D C$ & WHO & $\begin{array}{c}p \\
\text { value }\end{array}$ & $\mathrm{CDC}$ & WHO & $\begin{array}{c}p \\
\text { value }\end{array}$ \\
\hline Boys & \multicolumn{3}{|c|}{$n=381$} & \multicolumn{3}{|c|}{$n=416$} & \multicolumn{3}{|c|}{$n=407$} \\
\hline BMI percentile, mean (SD) & $53.8(31.5)$ & $56.6(32.5)$ & $<0.0001$ & $57.6(29.1)$ & $60.1(29.9)$ & $<0.0001$ & $57.8(27.8)$ & $58.7(28.3)$ & $<0.0001$ \\
\hline BMI z score, mean (SD)* & $0.12(1.1)$ & $0.35(1.4)$ & $<0.0001$ & $0.26(1.0)$ & $0.43(1.2)$ & $<0.0001$ & $0.28(1.0)$ & $0.38(1.1)$ & $<0.0001$ \\
\hline Underweight & $25 \quad(6.6)$ & $20 \quad(5.2)$ & $<0.0001$ & $21 \quad(5.0)$ & $21 \quad(5.0)$ & 0.002 & $11 \quad(2.7)$ & $10 \quad(2.5)$ & 0.006 \\
\hline Normal & $264(69.3)$ & $251(65.9)$ & $<0.0001$ & $295(71.0)$ & $275(66.1)$ & 0.002 & $306(75.2)$ & $296(72.7)$ & 0.006 \\
\hline Overweight & $57(15.0)$ & $66(17.3)$ & $<0.0001$ & $54(13.0)$ & $73(17.5)$ & 0.002 & $48(11.8)$ & $65(16.0)$ & 0.006 \\
\hline Obese & $35 \quad(9.2)$ & $44(11.5)$ & $<0.0001$ & $46(11.0)$ & $47(11.3)$ & 0.002 & $42(10.3)$ & $36 \quad(8.8)$ & 0.006 \\
\hline Underweight & 31 (7.8) & $25 \quad(6.3)$ & 0.001 & 15 (3.7) & $17 \quad(4.3)$ & 0.04 & $13 \quad(2.8)$ & $13 \quad(2.8)$ & 0.04 \\
\hline Normal & $283(71.1)$ & $274(68.8)$ & 0.001 & $293(73.3)$ & $281(70.3)$ & 0.04 & 352 (75.9) & $340(73.3)$ & 0.04 \\
\hline Overweight & $48(12.1)$ & $62(15.6)$ & 0.001 & $53(13.3)$ & $64(16.0)$ & 0.04 & 73 (15.7) & 86 (18.5) & 0.04 \\
\hline Obese & $36 \quad(9.0)$ & $37 \quad(9.3)$ & 0.001 & 39 (9.7) & $38 \quad(9.5)$ & 0.04 & $26 \quad(5.6)$ & $25 \quad(5.4)$ & 0.04 \\
\hline \multicolumn{10}{|c|}{$\begin{array}{l}\text { Note: } \mathrm{BMI}=\text { body mass index, } \mathrm{CDC}=\text { Centers for Disease Control and Prevention, } \mathrm{SD}=\text { standard deviation, } \mathrm{WHO}=\text { World Health Organization. } \\
\text { *Distance (measured in standard deviations) from the mean } \mathrm{BMI} \text { of children of the same age and sex. } \\
\text { tPercentages may not add up to } 100 \% \text { owing to rounding. } \mathrm{CDC} \text { and } \mathrm{WHO} \text { both define underweight as } \mathrm{BMI}<5 \text { th percentile and normal as at or above the } 5 \text { th } \\
\text { percentile and below the } 85 \text { th percentile. CDC defines overweight as a BMI at or above the } 85 \text { th percentile and below the } 95 \text { th percentile, and obese as } \mathrm{BMI} \geq \\
95 \text { th percentile. WHO defines overweight as at or above the } 85 \text { th percentile and below the } 97.7 \text { th percentile, and obese as } \mathrm{BMI} \geq 97.7 \text { th percentile. }\end{array}$} \\
\hline
\end{tabular}


standard cut-offs used by either the WHO or the CDC for defining overweight and obesity. In general, the ability of BMI percentiles calculated using the WHO and CDC growth curves to discriminate unfavourable levels of cardiometabolic risk factors did not significantly differ from one

Table 4: Comparison of the areas under the curves between the CDC and WHO BMI percentiles to predict cardiometabolic risk

\begin{tabular}{|c|c|c|c|c|c|c|c|c|c|}
\hline \multirow[b]{3}{*}{ Risk factor } & \multicolumn{9}{|c|}{ Area under the curve } \\
\hline & \multicolumn{3}{|c|}{$\begin{array}{c}\text { 9-year-olds } \\
n=779\end{array}$} & \multicolumn{3}{|c|}{$\begin{array}{c}13 \text {-year-olds } \\
n=816\end{array}$} & \multicolumn{3}{|c|}{$\begin{array}{c}16-y e a r-o l d s \\
n=871\end{array}$} \\
\hline & $\mathrm{CDC}$ & WHO & $\begin{array}{c}p \\
\text { value* }\end{array}$ & $C D C$ & WHO & $\begin{array}{c}p \\
\text { value* }\end{array}$ & $\mathrm{CDC}$ & WHO & $\begin{array}{c}p \\
\text { value* }\end{array}$ \\
\hline \multicolumn{10}{|c|}{ Total cholesterol $\geq 4.4 \mathrm{mmol} / \mathrm{L}$} \\
\hline Boys & 0.58 & 0.58 & 0.3 & 0.53 & 0.54 & 0.3 & 0.62 & 0.62 & 1.0 \\
\hline Girls & 0.52 & 0.52 & 0.3 & 0.51 & 0.51 & 0.3 & 0.48 & 0.48 & 0.3 \\
\hline \multicolumn{10}{|c|}{ HDL cholesterol < $1.0 \mathrm{mmol} / \mathrm{L}$} \\
\hline Boys & 0.53 & 0.53 & 0.7 & 0.64 & 0.64 & 0.3 & 0.58 & 0.58 & 1.0 \\
\hline Girls & 0.69 & 0.69 & 0.6 & 0.70 & 0.70 & 0.05 & 0.51 & 0.51 & 0.3 \\
\hline \multicolumn{10}{|c|}{ LDL cholesterol $\geq 2.6 \mathrm{mmol} / \mathrm{L}$} \\
\hline Boys & 0.59 & 0.59 & 1.0 & 0.52 & 0.52 & 0.3 & 0.60 & 0.60 & 0.3 \\
\hline Girls & 0.55 & 0.55 & 1.0 & 0.54 & 0.54 & 1.0 & 0.55 & 0.55 & 1.0 \\
\hline \multicolumn{10}{|l|}{ Triglycerides $\geq 1.7 \mathrm{mmol} / \mathrm{L}$} \\
\hline Boys & 0.89 & 0.89 & 0.7 & 0.87 & 0.86 & 0.05 & 0.80 & 0.80 & 0.3 \\
\hline Girls & 0.83 & 0.83 & 0.3 & 0.61 & 0.61 & 0.6 & 0.55 & 0.54 & 0.05 \\
\hline Systolic blood pressure $†$ & \multicolumn{3}{|c|}{$n=772$} & \multicolumn{3}{|c|}{$n=811$} & \multicolumn{3}{|c|}{$n=870$} \\
\hline Boys & 0.61 & 0.61 & 0.3 & 0.63 & 0.63 & 0.05 & 0.64 & 0.64 & 0.3 \\
\hline Girls & 0.71 & 0.71 & 0.3 & 0.70 & 0.70 & 1.0 & 0.67 & 0.67 & 0.3 \\
\hline \multicolumn{10}{|l|}{ Diastolic blood pressure †‡ } \\
\hline Boys & 0.71 & 0.72 & 0.1 & - & - & - & 0.52 & 0.52 & 1 \\
\hline Girls & - & - & - & 0.53 & 0.53 & 0.7 & 0.61 & 0.61 & 0.1 \\
\hline Insulin§ & \multicolumn{3}{|c|}{$n=706$} & \multicolumn{3}{|c|}{$n=723$} & \multicolumn{3}{|c|}{$n=812$} \\
\hline Boys & 0.77 & 0.77 & 0.3 & 0.74 & 0.74 & 0.3 & 0.75 & 0.74 & 0.3 \\
\hline Girls & 0.77 & 0.77 & 1.0 & 0.69 & 0.69 & 1.0 & 0.73 & 0.73 & 0.3 \\
\hline Glucose $\geq 5.6 \mathrm{mmol} / \mathrm{L}$ & \multicolumn{3}{|c|}{$n=779$} & \multicolumn{3}{|c|}{$n=816$} & \multicolumn{3}{|c|}{$n=871$} \\
\hline Boys & 0.65 & 0.65 & 0.6 & 0.57 & 0.57 & 0.3 & 0.49 & 0.49 & 1.0 \\
\hline Girls & 0.66 & 0.66 & 0.1 & 0.57 & 0.57 & 0.3 & 0.56 & 0.56 & 1.0 \\
\hline \multicolumn{10}{|c|}{ Cardiometabolic abnormalities } \\
\hline \multicolumn{10}{|l|}{1} \\
\hline Boys & 0.51 & 0.51 & 0.3 & 0.49 & 0.49 & 1.0 & 0.42 & 0.42 & 1.0 \\
\hline Girls & 0.53 & 0.53 & 0.3 & 0.47 & 0.47 & 1.0 & 0.44 & 0.44 & 1.0 \\
\hline \multicolumn{10}{|l|}{2} \\
\hline Boys & 0.67 & 0.66 & 0.6 & 0.60 & 0.60 & 0.3 & 0.52 & 0.52 & 1.0 \\
\hline Girls & 0.66 & 0.66 & 1.0 & 0.56 & 0.56 & 1.0 & 0.62 & 0.62 & 1.0 \\
\hline \multicolumn{10}{|l|}{$\geq 3$} \\
\hline Boys & 0.88 & 0.88 & 0.8 & 0.76 & 0.76 & 0.6 & 0.78 & 0.78 & 0.3 \\
\hline Girls & 0.84 & 0.84 & 0.3 & 0.78 & 0.78 & 0.3 & 0.76 & 0.76 & 0.3 \\
\hline \multicolumn{10}{|c|}{$\begin{array}{l}\text { Note: } \mathrm{BMI}=\text { body mass index, } \mathrm{CDC}=\text { Centers for Disease Control and Prevention, } \mathrm{HDL}=\text { high-density lipoprotein, } \mathrm{LDL}=\text { low-density lipoprotein, WHO = World } \\
\text { Health Organization. } \\
\text { *Reported } p \text { values are for the differences in the discriminative ability between the CDC and WHO growth curves using a continuity correction to account for the } \\
\text { non-independence of the observations. } \\
\text { tUnfavourable blood pressure } \geq 90 \text { th percentile for age, height and sex as defined by the National High Blood Pressure Education Program. } \\
\text { fThere were too few cases of unfavourable diastolic blood pressure ( } \geq 90 \text { th percentile) for } 9 \text {-year-old girls and } 13 \text {-year-old boys to calculate areas under the curve. } \\
\text { SInsulin } \geq 38 \text { pmol/L ( } 9 \text {-year-olds) or } \geq 60 \text { pmol/L ( } 13 \text { - and } 16 \text {-year-olds). } \\
\text { IVersus no abnormalities. Missing values were assumed to be normal. }\end{array}$} \\
\hline
\end{tabular}


another. This was true whether the areas under the curves were good or uninformative (Figure 1). Furthermore, although the differences in the areas under the curves between the WHO and CDC growth curves were marginally statistically significant for systolic blood pressure and

Table 5: Optimal BMI percentile cut-offs, as determined using the Youden index, for the CDC and WHO growth curves to predict cardiometabolic risk

\begin{tabular}{|c|c|c|c|c|c|c|}
\hline \multirow[b]{3}{*}{ Risk factor } & \multicolumn{6}{|c|}{ Area under the curve } \\
\hline & \multicolumn{2}{|c|}{$\begin{array}{c}\text { 9-year-olds } \\
n=779\end{array}$} & \multicolumn{2}{|c|}{$\begin{array}{c}\text { 13-year-olds } \\
n=816\end{array}$} & \multicolumn{2}{|c|}{$\begin{array}{c}16-y e a r-o l d s \\
n=871\end{array}$} \\
\hline & CDC & WHO & $\mathrm{CDC}$ & WHO & $C D C$ & WHO \\
\hline \multicolumn{7}{|c|}{ Total cholesterol $\geq 4.4 \mathrm{mmol} / \mathrm{L}$} \\
\hline Boys & 55.97 & 56.32 & 87.83 & 90.90 & 72.88 & 75.14 \\
\hline Girls & 65.09 & 68.86 & 35.60 & 34.16 & 39.92 & 36.41 \\
\hline \multicolumn{7}{|c|}{ HDL cholesterol $<1.0 \mathrm{mmol} / \mathrm{L}$} \\
\hline Boys & 61.30 & 64.69 & 42.96 & 45.60 & 78.66 & 80.91 \\
\hline Girls & 64.95 & 69.28 & 84.52 & 87.13 & 67.36 & 64.62 \\
\hline \multicolumn{7}{|c|}{ LDL cholesterol $\geq 2.6 \mathrm{mmol} / \mathrm{L}$} \\
\hline Boys & 53.18 & 56.32 & 87.83 & 90.90 & 72.61 & 74.95 \\
\hline Girls & 68.60 & 73.46 & 37.51 & 34.16 & 72.70 & 71.72 \\
\hline \multicolumn{7}{|l|}{ Triglycerides $\geq 1.7 \mathrm{mmol} / \mathrm{L}$} \\
\hline Boys & 87.17 & 91.76 & 90.84 & 94.80 & 76.07 & 78.15 \\
\hline Girls & 85.65 & 89.55 & 60.90 & 58.47 & 81.12 & 82.64 \\
\hline Systolic blood pressure* & \multicolumn{2}{|c|}{$n=772$} & \multicolumn{2}{|c|}{$n=811$} & \multicolumn{2}{|c|}{$n=870$} \\
\hline Boys & 48.51 & 34.68 & 67.21 & 71.54 & 86.17 & 88.63 \\
\hline Girls & 84.37 & 88.46 & 74.69 & 71.62 & 74.90 & 76.00 \\
\hline \multicolumn{7}{|l|}{ Diastolic blood pressure ${ }^{*} \dagger$} \\
\hline Boys & 96.01 & 99.0 & - & - & 35.67 & 36.21 \\
\hline Girls & - & - & 75.12 & 76.15 & 92.61 & 95.54 \\
\hline Insulin $\ddagger$ & \multicolumn{2}{|c|}{$n=706$} & \multicolumn{2}{|c|}{$n=723$} & \multicolumn{2}{|c|}{$n=812$} \\
\hline Boys & 83.41 & 88.16 & 84.91 & 88.36 & 80.96 & 83.58 \\
\hline Girls & 50.96 & 54.48 & 64.37 & 65.14 & 57.98 & 55.27 \\
\hline Glucose $\geq 5.6 \mathrm{mmol} / \mathrm{L}$ & \multicolumn{2}{|c|}{$n=779$} & \multicolumn{2}{|c|}{$n=816$} & \multicolumn{2}{|c|}{$n=871$} \\
\hline Boys & 87.43 & 91.96 & 65.43 & 68.27 & 27.05 & 27.31 \\
\hline Girls & 46.31 & 50.07 & 69.13 & 68.38 & 75.91 & 74.52 \\
\hline \multicolumn{7}{|c|}{ Cardiometabolic abnormalities§ } \\
\hline \multicolumn{7}{|l|}{1} \\
\hline Boys & 55.56 & 54.19 & 37.93 & 39.15 & 75.69 & 77.74 \\
\hline Girls & 65.09 & 69.35 & 66.34 & 69.09 & 75.73 & 75.77 \\
\hline \multicolumn{7}{|l|}{2} \\
\hline Boys & 54.38 & 64.39 & 62.76 & 67.59 & 72.61 & 74.95 \\
\hline Girls & 53.80 & 57.16 & 62.38 & 60.26 & 68.31 & 66.49 \\
\hline \multicolumn{7}{|l|}{$\geq 3$} \\
\hline Boys & 87.17 & 91.85 & 87.83 & 90.90 & 79.75 & 82.05 \\
\hline Girls & 85.21 & 89.24 & 71.56 & 68.42 & 72.74 & 71.72 \\
\hline \multicolumn{7}{|c|}{ 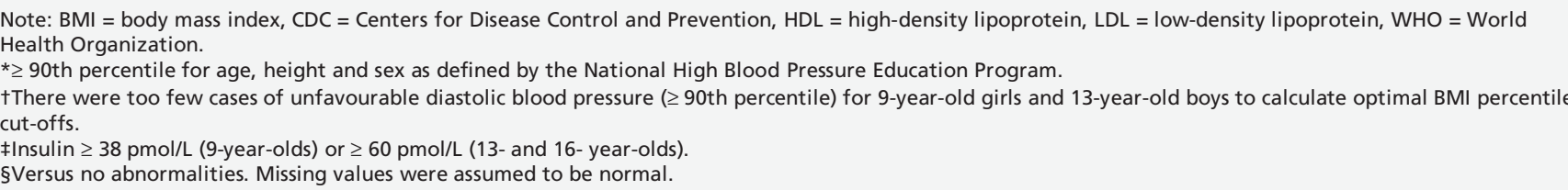 } \\
\hline
\end{tabular}




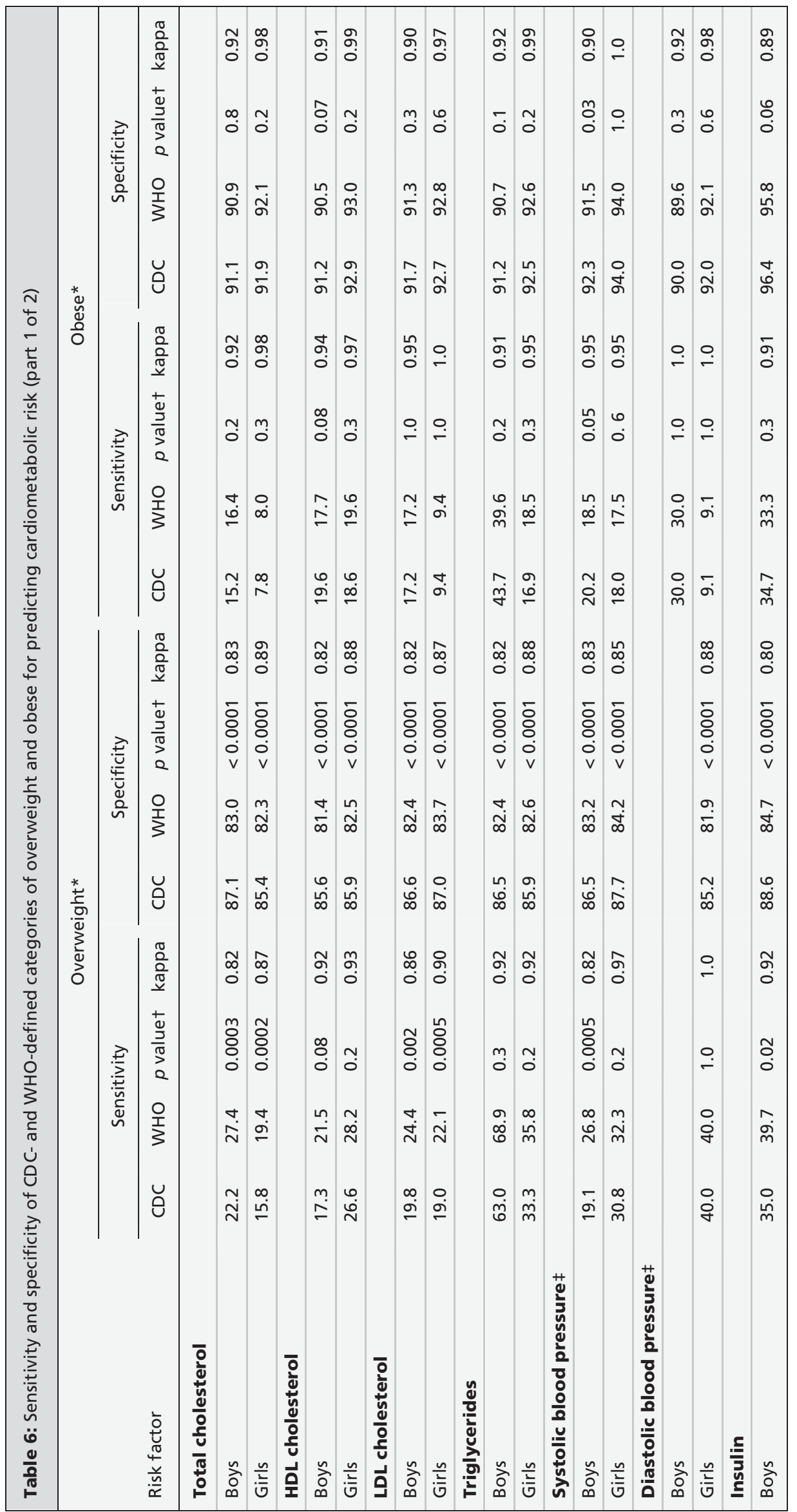




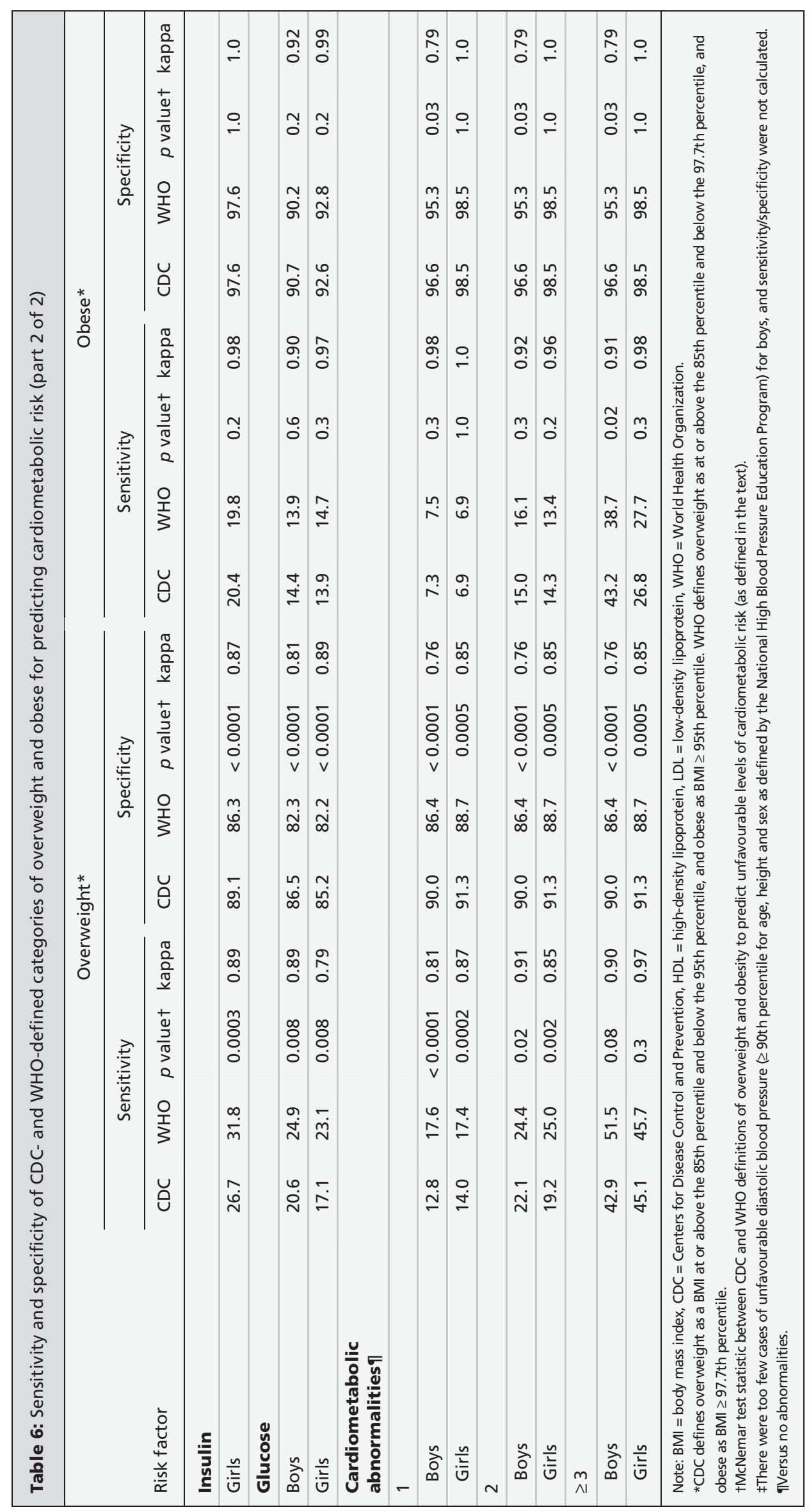


HDL cholesterol and triglyceride levels, they were not clinically meaningful.

Although the areas under the curves of the $\mathrm{CDC}$ and $\mathrm{WHO}$ growth curves were very similar, the sensitivity of the WHO-defined classification of overweight (compared with underweight or normal) was significantly higher than the CDC classification for many risk factors (Table 6). However, these improvements were small and ranged from $2.3 \%$ (95\% confidence interval [CI] $0.3 \%-3.2 \%$ ) to $8.6 \%$ (95\% CI $5.6 \%-9.6 \%$ ) for boys who were overweight, and from $0.6 \%$ (95\% CI $0.1 \%-0.8 \%$ ) to $6.0 \%$ (95\% CI $1.2 \%-6.7 \%$ ) for girls who were overweight. None met our acceptable sensitivity threshold of $70 \%$ (data not shown). The sensitivities of the obese classification were not significantly different between growth curves, and none met our acceptable sensitivity threshold. Both the CDC and WHO classifications of overweight and obese had acceptable specificities (> 70\%), although the specificities for the overweight classification as defined using the CDC growth curves were significantly higher than the specificities for the category as defined by the WHO. However, this improvement in specificity was small and ranged from $3.3 \%$ (95\% CI 3.0\%$4.0 \%$ ) to $4.2 \%$ (95\% CI $4.1 \%-4.9 \%$ ) for boys who were overweight and from $2.6 \%$ (95\% CI $1.2 \%-3 \%$ ) to $3.5 \%$ (95\% CI $2.4 \%-3.7 \%$ ) for girls who were overweight (data not shown). All kappa coefficients for sensitivity and specificity were greater than $75 \%$, suggesting good agreement between growth curves.

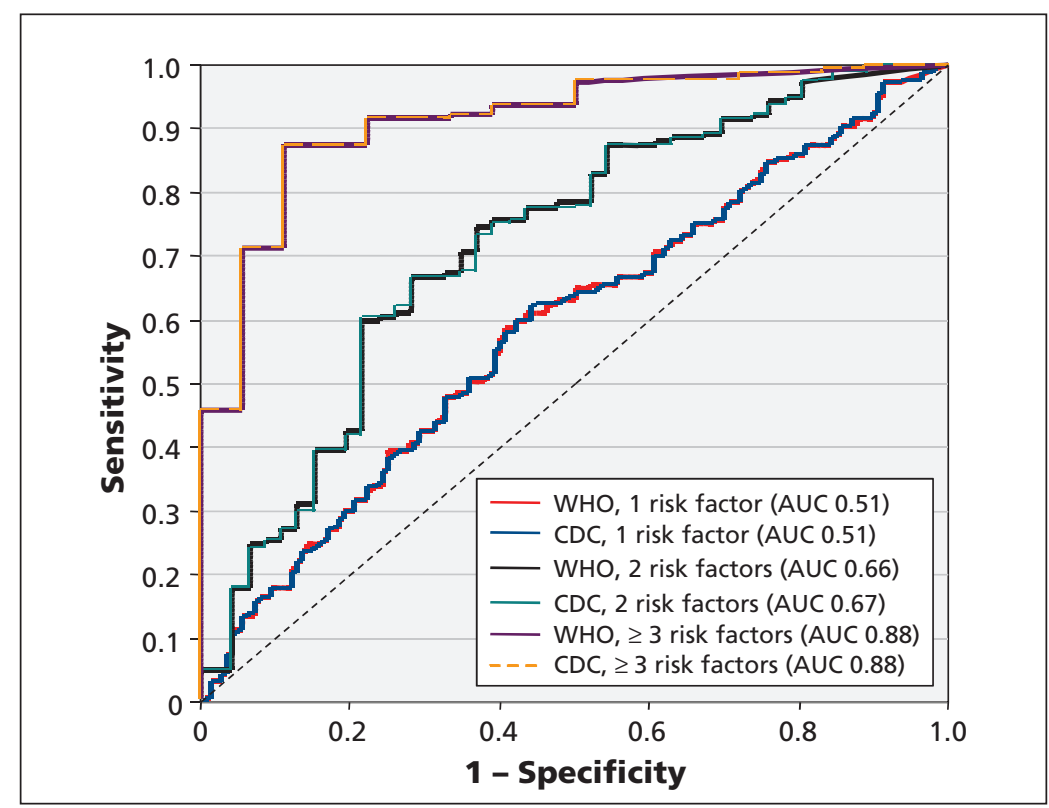

Figure 1: Receiver operating characteristic curves for detecting single or clustered (2, 3 or more) cardiometabolic risk factors among 9-year-old boys based on BMI percentiles calculated using the CDC and WHO growth curves. AUC = area under the curve, $\mathrm{BMI}=$ body mass index, $\mathrm{CDC}=$ Centers for Disease Control and Prevention, $\mathrm{WHO}=$ World Health Organization.

\section{Interpretation}

Although a given height and weight corresponds to different BMI percentiles on the CDC and WHO growth curves, the associations between BMI and cardiometabolic risk factors do not differ. This lack of difference is likely because most, but not all, of the data used to construct the $\mathrm{CDC}$ and $\mathrm{WHO}$ growth curves were drawn from the same reference populations. ${ }^{12,13}$

The American Heart Association and the American Academy of Pediatrics recommend lipid screening among young people who are overweight. ${ }^{7,8}$ Among the participants of our study, the WHO definition of overweight showed narrowly improved sensitivity over the CDC definition. Despite different percentile cut-offs for overweight and obese, both growth curves had specificities of more than $80 \%$, but sensitivities of less than $50 \%$. Thus, using overweight status to identify children with cardiometabolic risk will do so correctly among those who are overweight, but will poorly detect risk among those whose weight is classified as normal. The "optimal" BMI percentiles for detecting cardiometabolic risk covered a wide range, and the ability of BMI to predict risk among the participants in our study varied according to the specific cardiometabolic risk factors of interest.

\section{Limitations}

The CDC and WHO growth curves use BMI to measure fat mass, rather than dual-energy $\mathrm{x}$-ray absorptiometry, which is commonly viewed as the gold standard. However, BMI is noninvasive, inexpensive and well-validated for detecting cardiometabolic risk among young people. ${ }^{31,32}$

Previous studies suggest that the largest differences in $z$ scores between the CDC and WHO growth curves occur among young people with a BMI $z$ score of more than three standard deviations from the mean, but we could not assess this owing to the limited size of our sample.

We used data from a 1999 study involving 9-, 13- and 16-year-old children from Quebec, which may not be representative of other age groups or regions in Canada. However, our areas under the curves are similar to those reported in the Bogalusa Heart Study using CDC growth curves to assess a cohort of 5- to 17-year-old children from Louisiana. ${ }^{33}$ Because the intent of the QCAHS was to obtain a representative sample of children before, during and after the onset of puberty, our results may apply to adolescents of all ages.

Our study focuses on the present-day clinical impact of moving from one set of growth curves to another, but we cannot assess the ability of the 
growth curves to predict cardiovascular outcomes in adulthood. We did not collect data on the causes of cardiometabolic abnormalities such as hereditary dyslipidemia, but the impact on our results is likely small owing to the infrequency of such conditions.

Finally, we did not collect data on other surrogate measures of adiposity, such as waist circumference, and we cannot compare the relative utility of adding these measures to our analyses. However, there is no consensus on guidelines for waist circumference for young people. Furthermore, other surrogate measures have been found to be more prone to measurement error than height and weight, while only contributing minimal additional information to BMI. ${ }^{34,35}$

\section{Conclusion}

The WHO growth curves are recommended for monitoring growth in 5- to 19-year-old children because they use older data that precede the obesity epidemic, and they allow a smooth transition from the WHO growth curves recommended for monitoring growth in children aged $0-5$ years. ${ }^{14}$ However, our results suggest that the WHO growth curves do not add any discriminatory ad vantage over the $\mathrm{CDC}$ standards currently used in practice for the detection of cardiometabolic abnormalities among children aged $9-16$ years.

\section{References}

1. Freedman DS, Dietz WH, Srinivasan SR, et al. The relation of overweight to cardiovascular risk factors among children and adolescents: The Bogalusa Heart Study. Pediatrics 1999;103:1175-82.

2. Azita F, Asghar Z, Gholam-Reza S. Relationship of body mass index with serum lipids in elementary school students. Indian $J$ Pediatr 2009;76:729-31.

3. O'Malley G, Santoro N, Northrup V, et al. High normal fasting glucose level in obese youth: a marker for insulin resistance and beta cell dysregulation. Diabetologia 2010;53:1199-209.

4. Sinha R, Fisch G, Teague B, et al. Prevalence of impaired glucose tolerance among children and adolescents with marked obesity. N Engl J Med 2002;346:802-10. [Erratum in N Engl J Med 2002;346:1756.]

5. Ostchega Y, Carroll M, Prineas RJ, et al. Trends of elevated blood pressure among children and adolescents: data from the National Health and Nutrition Examination Survey 1988-2006. Am J Hypertens 2009;22:59-67.

6. Sorof J, Daniels S. Obesity hypertension in children: a problem of epidemic proportions. Hypertension 2002;40:441-7.

7. McCrindle BW, Urbina EM, Dennison BA, et al. Drug therapy of high-risk lipid abnormalities in children and adolescents: a scientific statement from the American Heart Association Atherosclerosis, Hypertension, and Obesity in Youth Committee, Council of Cardiovascular Disease in the Young, with the Council on Cardiovascular Nursing. Circulation 2007;115:1948-67.

8. Daniels SR, Greer FR. Lipid screening and cardiovascular health in childhood. Pediatrics 2008;122:198-208.

9. Khasnutdinova SL, Grjibovski AM. Prevalence of stunting, underweight, overweight and obesity in adolescents in Velsk district, north-west Russia: a cross-sectional study using both international and Russian growth references. Public Health 2010; 124:392-7.

10. Mei Z, Ogden CL, Flegal KM, et al. Comparison of the prevalence of shortness, underweight, and overweight among US children aged 0 to 59 months by using the CDC 2000 and the WHO 2006 growth charts. J Pediatr 2008;153:622-8.

11. Shields M, Tremblay MS. Canadian childhood obesity estimates based on WHO, IOTF and CDC cut-points. Int J Pediatr Obes 2010;5:265-73.
12. Kuczmarski RJ, Ogden CL, Guo SS, et al. 2000 CDC growth charts for the United States: methods and development. Vital Health Stat 11 2002;11:1-190.

13. de Onis M, Onyango AW, Borghi E, et al. Development of a WHO growth reference for school-aged children and adolescents. Bull World Health Organ 2007;85:660-7.

14. Dietitians of Canada, Canadian Paediatric Society, The College Of Family Physicians of Canada, Community Health Nurses of Canada. Promoting optimal monitoring of child growth in Canada: using the new WHO growth charts. A collaborative statement of the Dietitians of Canada, Canadian Paediatric Society, the College of Family Physicians of Canada, and Community Health Nurses of Canada. Can J Diet Pract Res 2010;71:e1-3.

15. Canadian Socio-Economic Information Management System (CANSIM). Population by sex and age group, 2010. Available: http://www40.statcan.gc.ca/101/cst01/demo10a-eng.htm (accessed 2012 Feb. 13)

16. Paradis G, Lambert M, O'Loughlin J, et al. The Québec Child and Adolescent Health and Social Survey: design and methods of a cardiovascular risk factor survey for youth. Can J Cardiol 2003; 19:523-31.

17. Tate JR, Rifai N, Berg K, et al. International Federation of Clinical Chemistry Standardization Project for the measurement of lipoprotein(a). Phase 1. Evaluation of the analytical performance of lipoprotein(a) assay systems and commercial calibrators. Clin Chem 1998;44:1629-40.

18. Tate JR, Berg K, Couderc R, et al. International Federation of Clinical Chemistry and Laboratory Medicine (IFCC) Standardization Project for the measurement of lipoprotein(a). Phase 2: selection and properties of a proposed secondary reference material for lipoprotein(a). Clin Chem Lab Med 1999;37:949-58.

19. Friedewald WT, Levy RI, Fredrickson DS. Estimation of the concentration of low-density lipoprotein cholesterol in plasma, without use of the preparative ultracentrifuge. Clin Chem 1972; 18:499-502.

20. Webber LS, Osganian V, Luepker RV, et al. Cardiovascular risk factors among third grade children in four regions of the United States. The Catch Study. Child and adolescent trial for cardiovascular health. Am J Epidemiol 1995;141:428-39.

21. Gidding SS, Dennison BA, Birch LL, et al. Dietary recommendations for children and adolescents: a guide for practitioners: consensus statement from the American Heart Association. Circulation 2005;112:2061-75. [Errata in Circulation 2005;112: 2375, Circulation 2006;113:e857.]

22. Lambert M, Delvin EE, Levy E, et al. Prevalence of cardiometabolic risk factors by weight status in a population-based sample of Quebec children and adolescents. Can J Cardiol 2008;24: 575-83.

23. National High Blood Pressure Education Program Working Group on High Blood Pressure in Children and Adolescents. The fourth report on the diagnosis, evaluation, and treatment of high blood pressure in children and adolescents. Pediatrics 2004; 114:555-76.

24. Lin WY, Lee LT, Chen CY, et al. Optimal cut-off values for obesity: using simple anthropometric indices to predict cardiovascular risk factors in Taiwan. Int J Obes Relat Metab Disord 2002; 26:1232-8.

25. Metz CE. Basic principles of ROC analysis. Semin Nucl Med 1978;8:283-98.

26. Youden WJ. Index for rating diagnostic tests. Cancer 1950;3:32-5.

27. Hanley JA, Hajian-Tilaki KO. Sampling variability of nonparametric estimates of the areas under receiver operating characteristic curves: an update. Acad Radiol 1997;4:49-58.

28. Hanley JA, McNeil BJ. A method of comparing the areas under receiver operating characteristic curves derived from the same cases. Radiology 1983;148:839-43.

29. Armitage P, Berry G. Statistical methods in medical research. Oxford (UK): Blackwell Scientific Publications; 1991.

30. Trajman A, Luiz RR. McNemar $\chi^{2}$ test revisited: comparing sensitivity and specificity of diagnostic examinations. Scand J Clin Lab Invest 2008;68:77-80.

31. Mei Z, Grummer-Strawn LM, Pietrobelli A, et al. Validity of body mass index compared with other body-composition screening indexes for the assessment of body fatness in children and adolescents. Am J Clin Nutr 2002;75:978-85.

32. Mei Z, Grummer-Strawn LM, Wang J, et al. Do skinfold measurements provide additional information to body mass index in the assessment of body fatness among children and adolescents? Pediatrics 2007;119:e1306-13.

33. Freedman DS, Kahn HS, Mei Z, et al. Relation of body mass index and waist-to-height ratio to cardiovascular disease risk factors in children and adolescents: the Bogalusa Heart Study. Am J Clin Nutr 2007;86:33-40.

34. Barlow SE. Expert committee recommendations regarding the 
prevention, assessment, and treatment of child and adolescent overweight and obesity: summary report. Pediatrics 2007;120 (Suppl 4):S164-92.

35. Freedman DS, Katzmarzyk PT, Dietz WH, et al. Relation of body mass index and skinfold thicknesses to cardiovascular disease risk factors in children: the Bogalusa Heart Study. Am J Clin Nutr 2009;90:210-6.

Affiliations: From the Department of Epidemiology, Biostatistics and Occupational Health (Kakinami, Henderson, Paradis), McGill University; the Department of Clinical Biochemistry (Delvin), the Department of Nutrition (Levy) and the Department of Pediatrics (Lambert), Centre Hospitalier Universitaire Sainte-Justine, Université de Montréal; Département de médecine sociale et préventive (O'Loughlin), Centre de recherche du Centre Hospitalier de l'Université de Montréal; and McGill University Health Centre Research Institute and the Institut national de santé publique du Québec (Paradis), Montréal, Que.

Contributors: Lisa Kakinami, Mélanie Henderson, Marie Lambert and Gilles Paradis conceived and designed the study. Lisa Kakinami analyzed the data and drafted the manuscript. All of the authors contributed to interpreting the data and critically revising the manuscript for important intellectual content. All of the authors approved the final version submitted for publication.

Funding: The Québec Child and Adolescent Health and Social Survey was funded by the Quebec Ministry of Health and Social Services and by Health Canada. The study on cardiovascular risk factors in young people was funded by the Canadian Institutes of Health Research (CIHR). Lisa Kakinami is supported through a CIHR grant (no. MOP-671210). Mélanie Henderson holds a doctoral research award from the CIHR. Jennifer O'Loughlin holds a Canada Research Chair in the Childhood Determinants of Adult Chronic Disease. Gilles Paradis holds an Applied Public Health Chair of the CIHR. The study sponsors had no role in the design of the study, the collection, analysis or interpretation of data, the writing of the report or the decision to submit the article for publication.

Acknowledgement: The authors thank Dr. James A. Hanley for his statistical input. 